\title{
Preferencias de Universidad y Carreras con mayor demanda en la Región San Martín 2017 - 2019
}

\author{
José Eber Paz Vilchez ${ }^{1}$, Kimberly López Díaz ${ }^{2}$, Lory Clarita Romero Chinguel ${ }^{3}$ \\ Escuela Profesional de Marketing y Negocios Internacionales, Universidad \\ Peruana Unión ${ }^{123}$
}

Recibido: 02 de noviembre de 2016

Aceptado: 05 de enero de 2017

\section{Resumen}

La presente investigación se desarrolló en el departamento de San Martin. El objetivo principal de este estudio es conocer las preferencias demandadas por los estudiantes al momento de elegir una carrera universitaria, así también la preferencia de la universidad para realizar sus estudios superiores. El método de estudio fue de tipo descriptivo, de diseño transversal. La población de estudio estuvo conformada por 1435 estudiantes de 14 a 20 años que vienen cursando del 3er año al 5to de secundaria la cual se tuvo como referencia las Provincias y Distritos de la Región San Martín. Tarapoto, Rioja, Moyobamba, Nueva Cajamarca, Juanjui, Yurimaguas, Bellavista Lamas y Tocache. Se aplicó el instrumento que se utilizó para medir las preferencias de las carreras más demandantes. Por las cuales se realizaron diferentes preguntas, basadas la elección de una carrera y el centro educativo donde estudiar. El resultado obtenido ubica en primer lugar a la Universidad Nacional de San Martín con un 25.6\%, (368), seguido por la Universidad Peruana Unión con un $18.8 \%$ (270), teniendo como carreras banderas medicina con 15.7\% (225), seguido por Ingeniería civil 14.4\% (217) y Administración de Empresas 11.2\% (161). Se concluye que en la Región de San Martín existe demanda por las Universidades y carreras tradicionales.

Palabras clave: Demanda, carreras universitarias, preferencias.

\footnotetext{
${ }^{1}$ Correspondencia al autor

E-mail: eberpaz@upeu.edu.pe
} 


\begin{abstract}
The present research was developed in the department of San Martin. The main objective of this study is to know the preferences demanded by the students at the time of choosing a university career, as well as the preference of the University for their higher education studies. The method of study was descriptive, cross-sectional design. The study population consisted of 1435 students from 14 to 20 years to come coursing in 3rd year to the 5th of secondary which took as a reference the Provinces and Districts of the Region San Martin. Tarapoto, Rioja, Moyobamba, New Cajamarca, Juanjuí, Yurimaguas, Bellavista Lamas and Tocache. The instrument was administered that was used to measure the preferences of the races more demanding. By which were performed different questions, based the choice of a career and educational center where to study. The result is obtained located in the first place to the National University of San Martin with a 25.6\%, (368), followed by the Universidad Peruana Union with a $18.8 \%$ (270), taking such as racing flags medicine with 15.7\% (225), followed by civil engineering 14.4\% (217) and Business Administration 11.2\% (161). It is concluded that in the region of San Martin demand exists for universities and traditional careers.
\end{abstract}

Keywords: Demand, university courses, preferences?

\title{
Introducción
}

Una de las preocupaciones en el campo educativo, la formación profesional de los estudiantes, básicamente en detectar en detectar toda la información referida a ideas, percepciones, y valoraciones sobre sus carreras, los planes de estudio, y las posibilidades de futuro. Es justamente en la institución universitaria donde se plasman, cobran vida y se cargan de sentido las representaciones de los estudiante. Leite (2015) refiere que las instituciones universitarias como lugar cúspide del saber dentro del sistema educativo formal, en el sentido de último nivel por el que transita un sujeto para alcanzar un título y su profesión. Al mismo tiempo, el estudiante llega un momento en que tiene que tomar decisiones, como es la elección de una carrera profesional y la institución que confía en toda su formación como profesional exitoso. Mesa-Sierra (2015) enfatiza sobre las preocupaciones, prejuicios, mitos e identificaciones que el estudiante al elegir posee de sí misma y del mundo que lo rodea, estos factores inherentes al proceso reflexivo que 
conducirá a una decisión acertada. Es decir a la obtención de un entendimiento o comprensión a través del dialogo o resolución del conflicto vocacional.

Algunos investigadores como De la Cruz y Mendoza (2010) realizaron un estudio similar titulado "Perfil Educativo Peruano 2010" teniendo como objetivo de estudio, conocer las preferencias de carreras, universidades, que manifiestan los estudiantes por estudiar en una institución educativa.

El objetivo principal del estudio es conocer las preferencias de las carreras universitarias al momento de hacer su elección, de la misma manera, conocer la preferencia de las universidades para realizar sus estudios superiores en la Región San Martín periodo 2017-2019.

\section{Investigación de mercados}

Malhotra (2008) afirma:

La investigación de mercados es la identificación, recopilación, análisis, difusión y uso sistemático y objetivo de la información con el propósito de mejorar la toma de decisiones relacionadas con la identificación y solución de problemas y oportunidades de marketing (p.48).

Para Zikmund y Babin, (2008) Investigación de mercados es:

La aplicación del método científico en la búsqueda de la verdad acerca de los fenómenos de marketing. Estas actividades incluyen la definición de oportunidades y problemas de marketing, la generación y evaluación de ideas, el monitoreo del desempeño y la comprensión del proceso de marketing. Es más que la mera aplicación de encuestas, este proceso incluye el desarrollo de ideas y teorías, la definición del problema, la búsqueda y acopio de información, el análisis de los datos y la comunicación de las conclusiones y sus consecuencias (p.6).

\section{Preferencias}

Para Pérez, Camarena, Cortazar y Góngora (2015) es el grado de aceptación e interés del individuo por la elección de un producto o servicio cubriendo sus necesidades.

Dado que el individuo en el papel de consumidor el centro y el creador de la cadena de demanda (Blackwell et al. 2002). Las elecciones de consumo están determinadas por dos factores, "posibilidades de consumo y preferencias", el primero está limitado por ingresos y los precios de los bienes y servicios que se compra y el segundo tiene relación con el beneficio o satisfacción que logra el consumidor. (Parkin, 2006, p. 176).

Según Avila (2004) para comprender el concepto de preferencia, es necesario rescatar elementos de origen microeconómico, relacionado al consumidor. La preferencia del consumidor, resulta un factor determinante de la demanda efectiva; que son los elementos subjetivos del consumidor que 
le hacen elegir determinados bienes y servicios que desea comprar. Para su análisis se consideran dos aspectos, disponibilidad de dinero para un determinado período de consumo y los precios de los bienes o servicios a comprar, que se expresa en la escala de preferencias. Existen una serie de factores que influyen en la preferencia del consumidor, como ser necesidades, gustos, caprichos, etc., que permitirán obtener satisfacción y utilidad por un período de tiempo. Además, el consumidor al tener información sobre el producto para su satisfacción y utilidad, y dado un nivel de ingreso, se concluye que está obligado comparar presupuestos y combinaciones, alternativas de bienes y servicios para determinar el orden de preferencia.

Afirman Schiffman y Kanuk (2005) que "La revolución de la información trajo consigo una serie de cambios, los consumidores tiene mayor poder sobre sus compras, mayor acceso a información para evaluar los productos, personalizar los productos y servicios, y comunicación interactiva entre ofertante y consumidor".

Según Juran (2005) el compromiso de los consumidores adecuadamente sensibilizados permitió poder evaluar un determinado producto con el fin de: desarrollar métodos objetivos para medir la preferencia del consumidor, como es el caso de las pruebas sensoriales y a partir de estos métodos se ha mejorado la toma de decisiones del productor orientado al control de procesos, ensayos de mercado y desarrollo, publicidad y comercialización de productos.

\section{Posicionamiento}

Es un elemento fundamental que mide el grado de ocupación de un producto y/o servicio en la mente de las personas y es el resultado de una estrategia especialmente diseñada para proyectar la imagen específica del producto, servicio, marca y hasta una persona con relación a la competencia. Kotler y Armstrong (2013) afirman "la forma en que un producto es definido por parte de los consumidores en sus atributos importantes con relación con los productos competidores” (p.182).

Caceres Carrasco y Vega Sepulveda (2008) afirman “el posicionamiento también se basa en la percepción, y la percepción es la verdad dentro del individuo. La percepción es el significado que da base a las experiencias, atributos, a los estímulos que nos entran por los sentidos”.

Becker y Kaldenberg (1993) confirman la importancia del posicionamiento por la relación que existe entre el éxito y crecimiento que la empresa pueda obtener y el estar bien posicionado en el mercado. Ahora bien, para lograr este posicionamiento las empresas se deben basar en una estrategia claramente definida. Las definiciones de estrategia son innumerables e incluso con 
diferencias de alcance, diversos autores coinciden en que la estrategia es la que guía la toma de decisiones y las relaciones entre la empresa y su entorno, afecta la estructura y los procesos dentro de la organización y como consecuencia los resultados que pueda obtener en su actividad.

Afirman Manu y Sriram (1996) que a nivel empresarial, la estrategia es sinónimo de orientación estratégica que comúnmente es definida como: la forma en la cual una organización usa la estrategia para adaptar o cambiar aspectos de su entorno a su favor. En una situación como la actual, caracterizada por la globalización y la internacionalización de los mercados, es fundamental para las empresas conseguir una posición competitiva fuerte sobre sus rivales, para ello es necesario elegir e implantar la estrategia con más probabilidades de éxito. La etapa de la formulación de la estrategia recoge la elección de una estrategia, esta elección no se puede hacer al azar o por intuición, para realizarla, es necesario tener un conjunto de posibles estrategias entre las cuales seleccionar una. Existe un abanico muy amplio de posibilidades para adquirir esa posición privilegiada, es decir, existen diferentes tipos de estrategias, ello se debe, fundamentalmente, a la gran cantidad de clasificaciones o tipologías de estrategias propuestas en la literatura, casi tantas como investigadores centrados en el campo de la estrategia empresarial.

Según García y Sabater (2003) existen diversas clasificaciones algunas de ellas se centran en el estudio de la estrategia a nivel de unidad de negocio, otros además de la unidad de negocio también estudian los distintos tipos de estrategias a nivel corporativo y otras clasificaciones, además de las dos anteriores tipologías, también recogen las formas de llevar a cabo estos dos niveles de estrategia: la estrategia corporativa es aquella que contempla a la empresa como un todo, aunque la empresa tenga distintos negocios en distintos sectores y la estrategia de negocio es aquella específica de cada una de las actividades llevadas a cabo por la empresa, sería el plan de actuación para cada negocio. En la estrategia a nivel de unidad de negocio, lo esencial es la construcción y sostenimiento de una ventaja competitiva, el fortalecimiento de esa ventaja competitiva a largo plazo será su objetivo esencial.

\section{Demanda}

Siguiendo la lógica de Best (2004), uno de los pocos investigadores que hablan de la orientación al mercado con sus componentes más amplios que incluyen lo criticado en líneas precedentes, define algunos de los conceptos fundamentales como: mercado potencial, demanda y cuota de 
mercado. Donde se deja entrever que los consumidores potenciales definen el nivel de la demanda futura, en mercados maduros el mercado potencial se define por el número máximo de clientes, pero que la demanda de mercado se define por el número de clientes existentes. Este autor remarca la importancia de entender: el mercado existente, el nivel de la demanda y la demanda potencial; asimismo, la tasa de una recompra y la compra de un nuevo cliente; incluyendo la cuota de mercado del negocio y la cuota del mercado potencial. Según el mismo, con la buena comprensión de estas dimensiones de desempeño se podrá evaluar el volumen actual y futuro del mercado, en otras palabras, el tamaño del mercado, y por consiguiente su demanda.

Entonces el concepto de mercado viene a ser crucial para el análisis de la demanda, sobre el que se desarrollaron teorías económicas muy importantes, el Diccionario de Economía y Finanzas de la EUMED (2004), en términos generales, señala que el mercado es el contexto dentro del cual toma lugar la compra y venta de mercancías, o donde se encuentran quienes demandan bienes y servicios con quienes los ofrecen. Aunque en castellano la palabra mercado designa frecuentemente el lugar físico donde se dan tales transacciones, el concepto económico es mucho más abstracto: se refiere al conjunto de interacciones humanas que, si bien tienen algún punto espacial de referencia, no deben por fuerza limitarse a un lugar determinado.

Kotler y Armstrong (2013) refiere que la demanda son deseos de un producto/servicio en función a una capacidad de acción determinada. Los deseos se transforman en demanda cuando existe capacidad adquisitiva, por lo que las empresas deben medir cuantas personas desean sus productos, y evaluar cuantos pueden adquirirlo potencialmente.

\section{Materiales y métodos}

El tipo de investigación que se ha utilizado es descriptivo, según Hernández, Fernández y Baptista (2014). Refiere que los estudios descriptivos tratan de especificar las características más importantes de un fenómeno, de esta manera se describen las tendencias y hechos ocurridos en un estudio determinado. La evaluación de cada una de las características, el tamaño representan los resultados que se pretende realizar para el logro del objetivo de estudio.

De diseño no experimental, según Hernández, Fernández y Baptista (2014) son estudios que se realiza sin la manipulación de variables, analizando los hechos ocurridos en la investigación, dada una investigación transversal permitiendo medir el estudio en un tiempo determinado. Los diseños 
transversales permiten periten indagar las modalidades o niveles de la investigación, se recolectan datos y se describe categoría, concepto, variables $\left(x_{1}\right)$, se recolectan datos y se describe categoría, concepto, variables $\left(x_{2}\right)$, se recolectan datos y se describe categoría, concepto, variables $\left(x_{3}\right)$, se recolectan datos y se describe categoría, concepto, variables $\left(x_{4}\right)$

\section{Participantes}

El estudio estuvo conformado por los estudiantes de ambos géneros del 3er, 4to y 5to año del nivel secundario en los Distritos y Provincias de la Región San Martín, teniendo como muestra. Tarapoto, Rioja, Moyobamba, Nueva Cajamarca, Juanjui, Yurimaguas, Bellavista Lamas y Tocache, teniendo como muestra representativa un total de 1435 estudiantes que independientemente participaron en el estudio. Entre las edades de 18 a 20 años.

\section{Tabla 1}

Provincias y Distritos más representativos de la Región San Martín en base a la institución educativa estatal y privada Adventista.

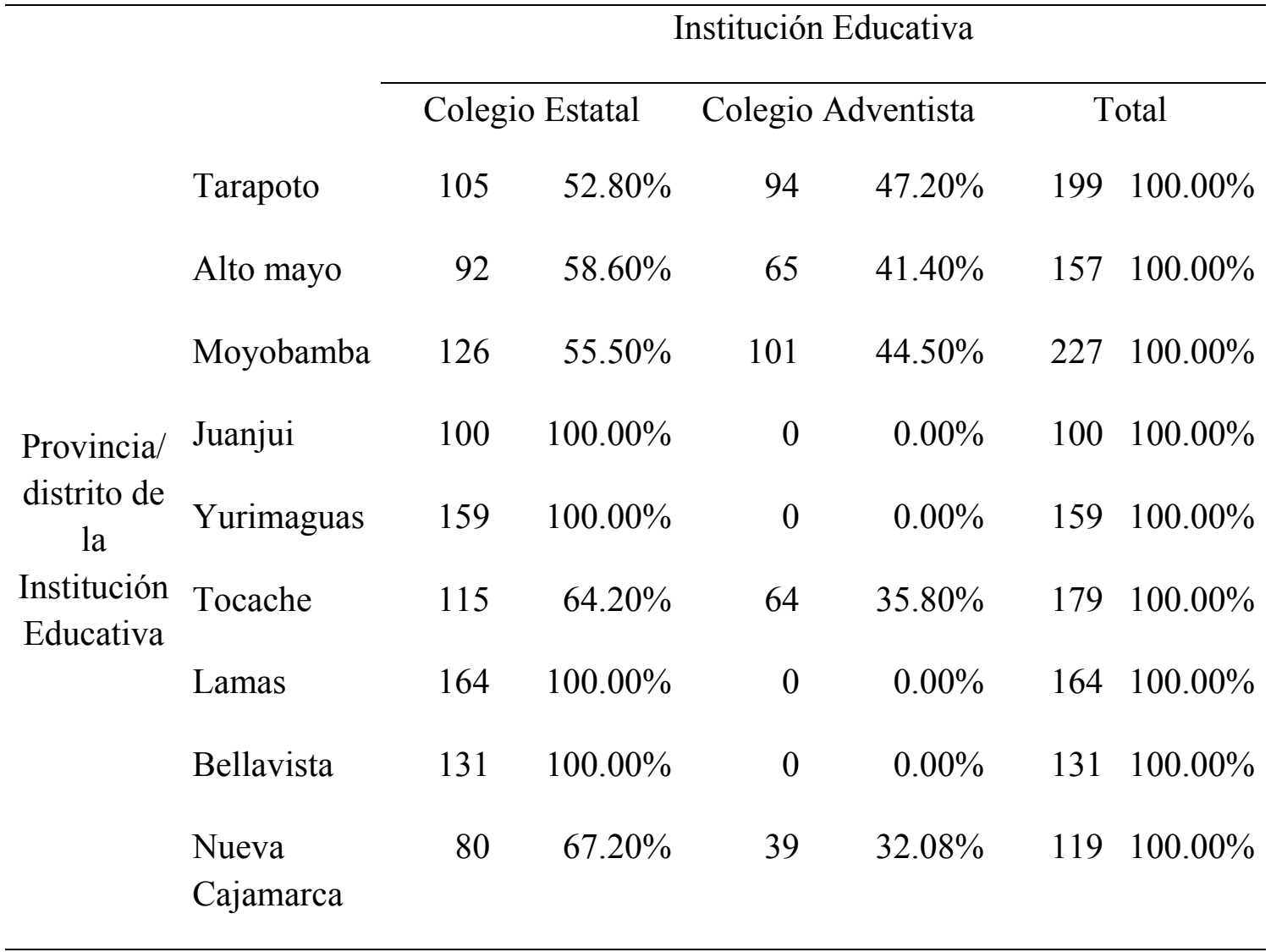


La tabla 01 muestra los diferentes distritos que se utilizaron en la muestra. Estos distritos pertenecen a la región de San Martín, Perú

\section{Instrumentos}

El instrumento para la recopilación de los datos fue el cuestionario, validado por juicio de expertos en el área de Marketing (Investigación de mercados), especialista en estadística, así mismo en la especialidad de Educación lingüística e inglés, estructurado en dos niveles, la primera se utilizó para la recopilación de datos generales, género, edad, provincia y/o distrito, religión, centro educativo, grado y sección. La segunda parte contiene 14 preguntas de selección, que ha permitido la descripción del estudio en general, obteniendo como preguntas básicas ¿Qué Carrera te gustaría estudiar en la Universidad Peruana Unión campus Tarapoto? Que ha justificado medir el grado de aceptación de las carreras actuales, de igual forma se preguntó ¿Qué carrera te gustaría estudiar en la Región San Martín?, permitiendo conocer las carreras con mayor demanda estudiantil para los años de 2017 al 2019.

\section{Análisis de Datos}

El procesamiento de los datos se realizó mediante el Programa Estadístico Informático del SPSS versión 23, aplicado al análisis de las preguntas y congruencias entre ellas, también se utilizó el programa del Excel permitiendo representar de manera gráfica y porcentual los resultados de la investigación. 


\section{Tabla 2}

Distribución de la muestra en colegios estatales y colegios adventistas

Institución Educativa

\section{Colegio Estatal \\ Colegio Adventista \\ Total}

\begin{tabular}{llrrccc}
\hline & $3^{\circ}$ & 199 & $77.70 \%$ & 57 & $22.30 \%$ & $100.00 \%$ \\
$\begin{array}{l}\text { Año de } \\
\text { estudio }\end{array}$ & $4^{\circ}$ & 351 & $80.00 \%$ & 88 & $20.00 \%$ & $100.00 \%$ \\
& $5^{\circ}$ & 522 & $70.50 \%$ & 218 & $29.50 \%$ & $100.00 \%$ \\
& Total & 1072 & $74.70 \%$ & 363 & $25.30 \%$ & $100.00 \%$ \\
\hline \hline
\end{tabular}

La muestra representativa estuvo conformada por el $77.70 \%$ que representan a los estudiantes del tercer año en el nivel secundario y el $22.30 \%$ a los estudiantes de colegios adventistas, $80.00 \%$ lo conforman los estudiantes del cuarto año del nivel secundario y el $20 \%$ de colegios adventistas, el $70.50 \%$ representa a los estudiantes del quinto año del nivel secundario y el $29.50 \%$ a $\operatorname{los}$ estudiantes de colegios adventistas. En general, la muestra que representa mayor número son los estudiantes del quinto año del de colegios estatales y adventistas.

Gráfico 1 Preferencia en Instituciones superiores para estudiar

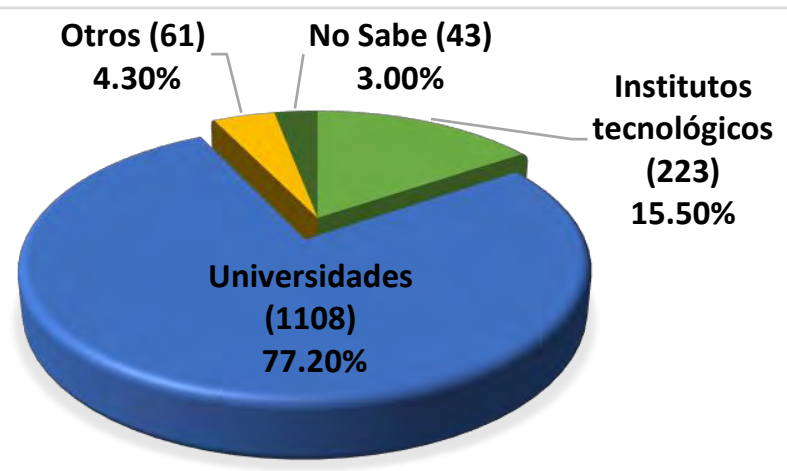


Los estudiantes del tercero, cuarto y quinto año del nivel secundario demuestran más preferencias por estudiar en universidades con un 77.20\%, representado en 1108 estudiantes, como segunda opción tienen preferencias para estudiar en institutos tecnológicos representando el $15.50 \%$, el $4.30 \%$ eligen otras instituciones, y el 3.00\% aún no deciden en que instituciones estudiar.

Gráfico 2 Preferencias de Universidades con mayor demanda para estudiar

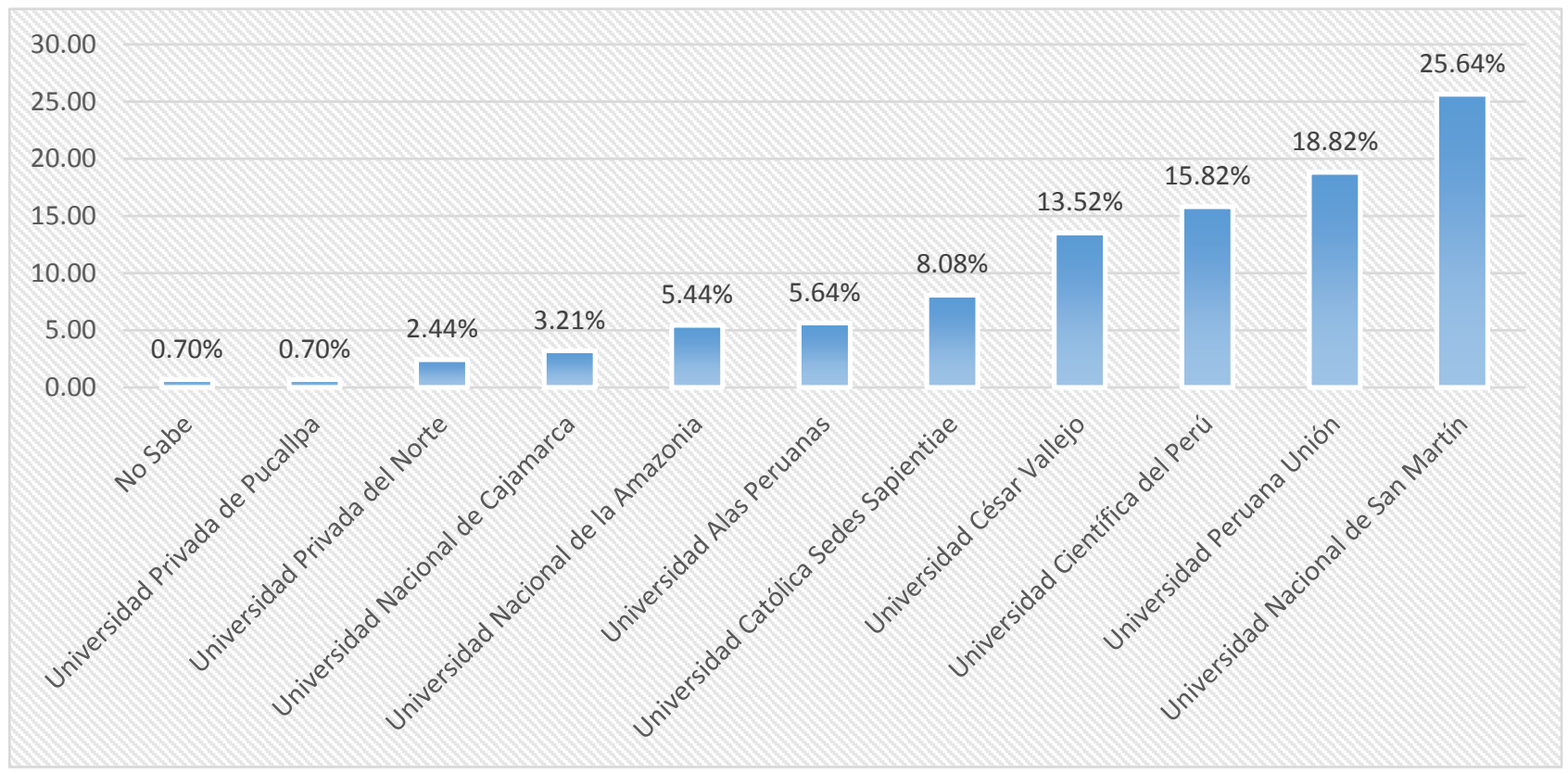

El gráfico 02 representa las universidades que tienen mayor preferencia en estudiar algunas careras por parte de los estudiantes en la Región San Martín, teniendo como resultados que el 25.4\% tienen preferencias por estudiar en la Universidad Nacional de San Martín, seguido por la Universidad Peruana Unión con 18.82\%, la Universidad Científica del Perú con 15.82\%, continuación la Universidad Cesar Vallejo con 13.52\%, la Universidad Católica Sede Sapientiae con $8.08 \%$, la Universidad Alas Peruanas que representa el 5.64\%, seguido por la Universidad Nacional de la Amazonía con un 5.44\%, y otras Universidades que no tienen presencia en la Región San Martín. 


\section{Gráfico 3}

Demanda estudiantil de las carreras actuales que tiene la Universidad Peruana Unión Filial Tarapoto.

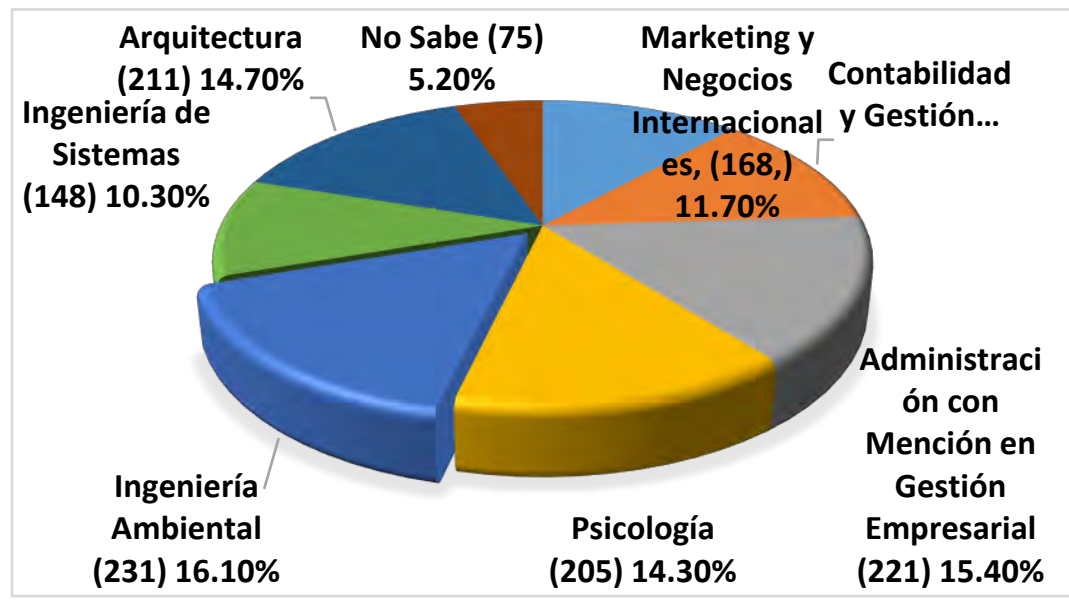

Se puede apreciar en el gráfico 03 la evaluación de preferencia sobre las carreras actuales que cuenta la Universidad Peruana Unión, obteniendo como resultado que la carrera más demandada es Ingeniería Ambiental que representa al 16.10\%, continua la carrera de Administración con Mención en Gestión Empresarial, representando el 15.40\%, seguido por la carrera de Psicología representando el $14.30 \%$, luego está la carrera de Arquitectura con $14.7 \%$, seguido por la carrera de Contabilidad y Gestión Tributaria que representa el 12.30\%, marketing y Negocios Internacionales representa el $11.70 \%$, Ingeniería de sistemas con $10.30 \%$, y el $5.20 \%$ que representa a 75 estudiantes no muestran ninguna opinión referente a las carreras actuales. 


\section{Gráfico 4}

Carreras con mayor demanda en la Región San Martín año 2017-2019.

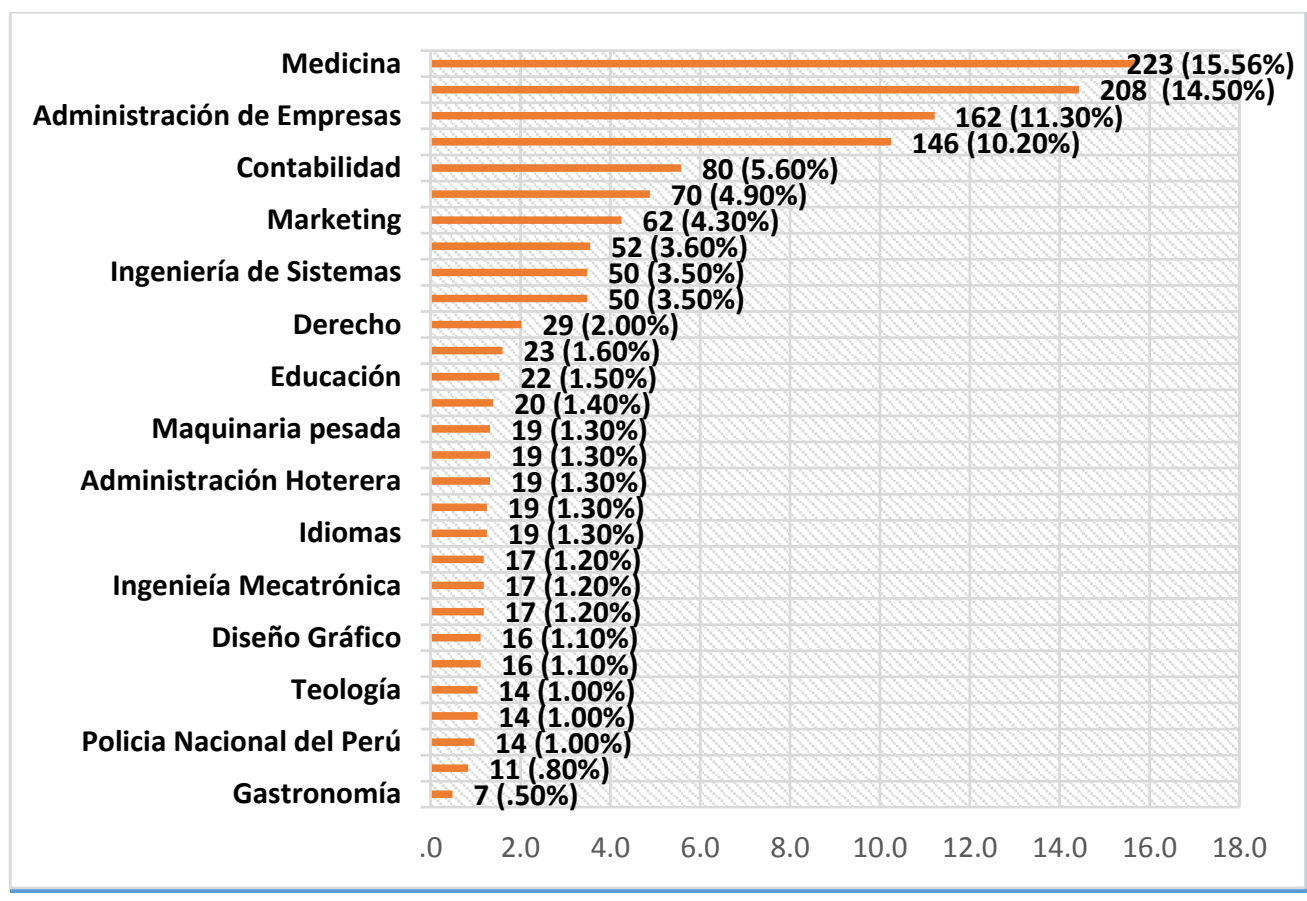

De acuerdo a la demanda de los estudiantes sobre las carreras preferenciales para estudiar, se ha obtenido como resultados que existe mayor demanda por la carrera de medicina, con un porcentaje mayor de $15.70 \%$, seguido por la carrera de Ingeniería Civil que representa el $14.40 \%$, continua la carrera, Administración de Empresas con 11.20\%, seguido por la carrera de Enfermería que representa el $10.20 \%$, también está la carrera de Contabilidad con 5.60\%, seguido por Ingeniería Ambiental con $4.90 \%$, continua la carrera de Marketing con $4.30 \%$, seguido por Arquitectura representando el 3.60\%, seguido por Ingeniera de Sistemas que representa el 3.50\%, de la misma marera la carrera de Administración y Negocios Internacionales con 3.50\%, continua la carrera de Derecho representando al grado de preferencia de $2.00 \%$. Continúan las carreras que tienen un bajo porcentaje de preferencias.

\section{Conclusiones}

De acuerdo a la preferencia del tipo de instituciones, los estudiantes consideran como primera alternativa realizar sus estudios superiores en las universidades, debido a la calidad y exigencias 
académicas, por algunas dificultades que se presenten, una mínima cantidad prefieren seguir estudiando en los institutos.

En cuanto a la preferencia de universidades en la Región San Martín, los estudiantes prefieren en primer lugar la institución estatal, Universidad Nacional de San Martín, luego están las universidades particulares. Al mismo tiempo se mide el posicionamiento que tienen las universidades.

Sobre las carreras actuales que cuanta la Universidad Peruana Unión se mantiene el grado de preferencias por estudiar, ya que forman parte de las carreras más demandas del mercado.

En las carreras con mayor demanda estudiantil están, medicina, ingeniería civil, administración de empresas, enfermería, contabilidad, ingeniería ambiental, marketing, arquitectura, ingeniería de sistemas, administración y negocios internacionales, y la carrera de derecho. Tal resultado demuestra que los estudiantes tienen el anhelo de continuar sus estudios con una carrera profesional.

\section{Recomendaciones}

a) Realizar el seguimiento con la base de datos y resultados de la investigación, para informarles a los estudiantes que puedan estudiar sus universidades y la preferencia de sus carreas.

b) Se recomienda analizar las condiciones socioeconómicas de los estudiantes por regiones y/o distritos

c) Es conveniente realizar nuevas investigaciones para medir el posicionamiento De las universidades, y las nuevas demandas de los estudiantes.

d) Realizar las universidades campañas de publicitarias para la captación de nuevos ingresantes

e) Mediante esta investigación se recomienda implementar nuevas carreras que estén de acorde con las exigencias del mercado estudiantil. 


\section{Referencias}

Ávila, J. (2004). Introducción a la economía. México: Plaza y Valdés, S.A. de C.V.

Blackwell, Miniard, \& Engel (2002). Comportamiento del consumidor. México: International Thompson Editores S.A. de C.V.

Caceres Carrasco, J. y Vega Sepulveda, C. (2008). Posicionamiento y Determinación del Mercado Objetivo Potencial de Dimarsa S . A . Tesis de grado no publicado Universidad Austral de Chile.

Cruz, I. (1995). Fundamentos del marketing. Barcelona: Editorial Ariel.

De la Cruz, A. \& Mendoza, H. (2010). Perfil educativo peruano 2010. Apunt. Univ. 1(1), 107-118.

García, M. y Sabater, R. (2003). Relaciones entre estrategia y ciclo de vida de la empresa. Revista Madrid No. 20.

García, R. (2003) Motivos de elección de carrera en las profesiones de alta y baja demanda. Tesis de maestria no publicada. Universidad Autónoma de Nuevo León.

Kotler, P. (1985) Mercadotecnia. Mexico: Prentice hall

Kotler, P. \& Armstrong, G. (2013). Fundamentos de Marketing. México: Pearson Educación.

Lado Couste, N. (1995). Orientacion al mercado y resultados economicos. Tesis doctoral no publicada. Universidad Autonoma de Madrid.

Leite, E. (2015). Las representaciones de las carreras universitarias desde la mirada de los estudiantes. Iberoamericana de Educación. 39(3), 1-6.

Malhotra, N. (2008). Investigación de Mercados. Mexico. Pearson Educación.

Mesa-Sierra, G. (2015). Diferencias de género en percepción y preferencia de carrera universitaria Graciela Meza Sierra. Tecnología En Marcha 13(2), 10-20.

Orozco, L. (2009) Variables que inciden en la elección de carrera y casa de estudios, en la perspectiva del género. Tesis de grado no publicada. Universidad de Chile.

Uzcanda, N., Camarena, D., Cortazar, M. y Góngora, R. (2015). Preferencias de consumo por productos derivados del cocotero en la Península de Yucatán, México. Revista Mexicana de Ciencias Agricolas, 6(1), 45-47.

Villarejo, A. (2001) La medición del valor de marca los efectos del esfuerzo de marketing sobre sus componentes. Tesis doctoral no publicada. Universidad de Sevilla. 
Zikmund, W. y Babin, B. (2008). Investigación de Mercados. Cengage Learning Editores. S.A. de C.V. 